\section{Condições associadas a prejuízo de desempenho em habilidades sociais em uma amostra de conveniência de usuários de crack}

\section{Conditions associated with impaired social skills in a convenience sample of crack users}

\author{
Condiciones asociadas a prejuicios sobre el \\ desempeño en habilidades sociales dentro de \\ una muestra de conveniencia con \\ consumidores de crack
}

Rogério Lessa Horta 1 Julia Luiza Schäfer 1 Leda Rubia Maurina Coelho 2 Viviane Samoel Rodrigues 3 Margareth Silva de Oliveira 2 Vanessa Andina Teixeira 1

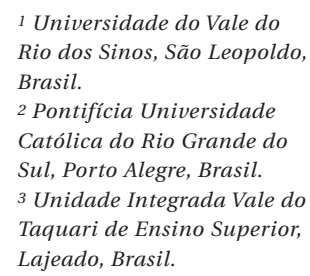
Rio dos Sinos, São Leopoldo, Brasil.

2 Pontifícia Universidade Católica do Rio Grande do Sul, Porto Alegre, Brasil. 3 Unidade Integrada Vale do Taquari de Ensino Superior, Lajeado, Brasil.

\author{
Correspondência \\ R. L. Horta \\ Rua Jari 671, apto. 703, Porto \\ Alegre, RS 91350-170, Brasil. \\ rogeriohortamed@gmail.com
} amostragem de conveniência de 519 indivíduos, entrevistados em Escore deficitário no IHS ocorreu para 52,8\% da amostra e não houv ferenças quanto a estar ou não em uso ativo de crack, ou quanto a estc ou não em atendimento. Apresentaram probabilidade aumentada de déficit no IHS os usuários de crack em pré-contemplação ou contemplação no University of Rhode Island Change Assessment - URICA ( $p=0,031)$, com escores elevados no Inventário de Depressão de Beck - BDI $(p=0,037)$ os que viveram luto de filho ( $p=0,001)$ e os que já assaltaram para obter $o$ crack $(p=0,020)$. Com probabilidade diminuida estavam os que tinham escolaridade de 9 anos ou mais ( $p=0,001)$, apoio social elevado $(p<0,001)$, resiliência elevada $(p<0,001)$, com histórico de ter perdido o contato com seus pais ( $p=0,032)$, já tendo sido hospitalizado em função da droga ( $p=0,009)$ e que relataram já ter mantido atividade sexual para obter a pedra ( $p=0,009)$. Os achados têm utilidade clínica e mostram a importância do tema.

Habilidades Sociais; Crack; Cocaína; Inteligência Emocional;

Transtornos Relacionados ao uso de Substâncias 


\section{Introdução}

O termo "habilidades sociais" tem sido utilizado para nominar capacidades comportamentais aprendidas que envolvem interações sociais 1 . Trata-se de um conjunto de comportamentos que demonstram a habilidade do indivíduo em expressar sentimentos, anseios e opiniões adequadamente ao que cada situação exige, conseguindo, deste modo, resolver problemas e conflitos 2 . Ainda podem ser vistas como diferentes classes de comportamentos sociais do sujeito para responder de modo adequado às demandas de situações oriundas dos relacionamentos interpessoais 3 .

Desempenho rebaixado em termos de habilidades sociais pode estar associado a comportamentos disfuncionais como o uso abusivo de álcool e outras drogas 4. A busca por substâncias psicoativas pode ser vista como uma solução ou como um paliativo para situações de sofrimento, quando há indisponibilidade de outros recursos, entre eles, um repertório mais amplo de habilidades sociais 5 e, de outro modo, o déficit em habilidades sociais parece estar relacionado à dificuldade em resistir às drogas e dizer não 6,7 .

Na literatura atual, alguns estudos apresentam relações entre habilidades sociais e comportamentos relacionados ao uso abusivo de álcool e outras drogas. O Inventário de Habilidades Sociais (IHS) tem sido empregado, entre outros temas de interesse, na prospecção de condições associadas ao uso de substâncias psicoativas ou aos padrões de resposta obtidos em modalidades terapêuticas propostas a indivíduos ou grupos de pessoas com problemas decorrentes do consumo de álcool e de outras drogas 8,9,10. Os repertórios de autocontrole da agressividade em situações aversivas e de autoexposição a desconhecidos e situações novas de adolescentes dependentes ou abusadores de maconha foram menores em comparação aos dos não usuários da droga 8 . Além disso, outro estudo identificou que tabagistas apresentam maior ansiedade social, comparados a não tabagistas, em duas dimensões: interagir com desconhecidos e ser o centro das atenções 9. Da mesma forma em que estudos identificam essa relação, um trabalho realizado no Brasil não observou diferença nos escores do IHS entre dependentes e não dependentes de álcool 10. Os autores admitem problemas metodológicos nesse trabalho.

Além da avaliação do repertório de habilidades, a utilização de técnicas de psicoterapia envolvendo o treinamento de habilidades sociais é citada para o tratamento dos dependentes de drogas 11,12,13,14. Smith et al. 15, porém, não encontraram diferença ao reproduzirem com adolescentes um estudo realizado com adultos americanos afrodescendentes, usuários de álcool, que receberam treinamento de habilidades como parte do tratamento para uso de substâncias.

Entre as diferentes substâncias psicoativas disponíveis, o crack tornou-se um problema em saúde pública nas últimas décadas e tem demonstrado seu poder de promover desfechos em saúde com graves consequências 16,17,18. A criminalidade é um exemplo de decorrência preocupante do uso de $\mathrm{crack}$, frequentemente referida ${ }^{19}$. Dualibi et al. 16 afirmam haver evidências de que a deficiência em habilidades sociais é um dos fatores preditivos para o abandono do tratamento da dependência de cocaína e crack.

Inúmeras condições associadas ou determinantes do consumo de cocaína ou crack podem também estar associadas a escores deficitários em termos de habilidades sociais, e esta relação pode produzir distorções em análises relativas à associação entre habilidades sociais e resposta a diferentes modalidades de tratamento em transtornos por uso de drogas. Uma questão relevante ao considerar essas respostas a diferentes tratamentos é o número significativo de indivíduos que buscam por tratamento para uso de substâncias e desistem prematuramente, ou recaem logo após o seu término. Uma condição associada a esses dados é uma sensibilidade à ansiedade, ou seja, o medo da sensação de ansiedade originado pela crença de que esta pode trazer consequências prejudiciais. Essa condição aparece fortemente associada a formas desadaptativas de processamento emocional e regulação do afeto, como uso de estratégias de evitação 20 , aspecto associado a déficits em habilidades sociais 21 .

O estudo de habilidades sociais entre usuários de substâncias como o crack pode oferecer contribuições relevantes, tanto no sentido de qualificar os modelos terapêuticos propostos, como no de indicar aspectos a serem contemplados em ações ou programas de prevenção de agravos quando se pensa em populações que já apresentam o comportamento de uso da droga.

O objetivo deste trabalho é investigar as condições associadas a escores deficitários no IHS entre usuários de crack da Região Metropolitana de Porto Alegre, Rio Grande do Sul, Brasil.

\section{Método}

Trata-se de um estudo transversal, com caráter exploratório, realizado em 2011. A amostra estudada envolveu 519 usuários de crack da Região Metropolitana de Porto Alegre. A amostragem foi realizada por conveniência, pelo processo bola 
de neve, em que os entrevistados ou os profissionais dos serviços de saúde indicavam indivíduos para participarem da pesquisa. Foram visitados, como pontos iniciais de busca de entrevistados, sete clínicas privadas, seis Centros de Atenção Psicossocial (CAPS), um serviço comunitário de atendimento e orientação a usuários e familiares, um hospital, dez comunidades terapêuticas e duas casas de passagem mantidas por comunidades terapêuticas para acolhimento de usuários. Alguns poucos entrevistados identificados em espaços públicos concordaram em participar do estudo.

Foram incluídos na amostra todos os indivíduos que informaram ter usado crack alguma vez na vida. Ainda assim, o grupo de entrevistados não incluiu pessoas que tenham feito uso eventual, uma única vez, há muito tempo. O processo de indicação por pessoas-chave e a busca com base em centros de atendimento levou a equipe do estudo ao contato com indivíduos reconhecidos por pessoas próximas e por eles mesmos como usuários de crack, alguns deles já com uso cessado por algum tempo.

Entre os entrevistados, é possível reconhecer diferentes intervalos de tempo entre o último episódio de consumo do crack e o momento da entrevista. O grupo de usuários que referiu o último episódio de consumo há 12 semanas ou mais no momento da entrevista foi considerado em abstinência, por já se caracterizar cessação do comportamento de consumo mantida, além de ser este o intervalo indicado como de risco mais elevado de recaída 22.

Considerou-se critérios de exclusão quaisquer condições que levassem à incapacidade objetiva de responder ao questionário, manifestada pelo entrevistado ou detectada pelo entrevistador, independentemente da incapacidade ser temporária ou persistente.

A coleta de dados foi realizada por entrevistadores selecionados e treinados previamente. Foi empregado um questionário desenvolvido pela equipe, pré-codificado, padronizado e prétestado, ao qual foram integrados alguns instrumentos validados. Os questionários foram codificados e digitados em sistema de dupla entrada no programa SPSS versão 17.0 (SPSS Inc., Chicago, Estados Unidos). Foram checados 5\% do total das entrevistas para controle de qualidade do preenchimento dos dados.

Este estudo foi aprovado pelo Comitê de Ética em Pesquisa da Universidade do Vale do Rio dos Sinos, segundo resolução CEP/Unisinos no 027/2010, de 22/Mar/2010, e financiado pelo Conselho Nacional de Desenvolvimento Científico e Tecnológico (CNPq) no Edital 14/2009 Universal, processo MCT/CNPq 476941/2009-1.
O desfecho deste estudo é o escore no IHS 23 igual ou inferior ao percentil 36, definido como escore deficitário. Trata-se de um instrumento que avalia as habilidades sociais, indicando o desempenho social do indivíduo em diferentes situações/áreas de vida. Os escores fatoriais estão divididos da seguinte forma: (1) habilidades de enfrentamento com risco; (2) habilidades de autoafirmação na expressão de afeto positivo; (3) habilidades de conversação e desenvoltura social; (4) habilidades de autoexposição a desconhecidos ou a situações novas; (5) habilidades de autocontrole da agressividade em situações aversivas.

O modelo de análise utilizado propõe que até a ocorrência do desfecho existe uma cadeia de determinantes hierarquizados em que os níveis acima determinam os níveis abaixo na cadeia, dispostos como ilustrado na Figura 1, e todos determinam o comportamento do desfecho.

Foram incluídas no modelo de análise, no nível distal, as variáveis demográficas e socioeconômicas investigadas: sexo, idade, escolaridade, situação conjugal, ter ocupação regular, renda e composição do domicílio (número de pessoas em casa).

No nível intermediário foram incluídos fatores psicossociais, como a ocorrência de estressores psicossociais nos 12 meses anteriores às entrevistas e escores de resiliência e apoio social 24, além das variáveis relacionadas ao uso do crack, como a condição de uso no momento e uso compartilhado com familiares ou amigos.

No nível proximal, ingressaram no modelo as variáveis relacionadas a tratamentos realizados ou desejados pelos entrevistados; sua condição clínica no momento das entrevistas, como o estágio de prontidão para a mudança, aferido pelo URICA (University of Rhode Island Change Assessment) validado para o Brasil 25; autopercepção de saúde; níveis de ansiedade e depressão na data da entrevista, aferidos pelo BAI (Inventário de Ansiedade de Beck); BDI (Inventário de Depressão de Beck) 26,27, inventário de critérios para dependência de crack 28 e registro de danos percebidos como decorrentes do uso da droga, inclusive esforços para obtê-la considerados prejudiciais.

Foi realizada análise descritiva para a obtenção da distribuição de frequências das categorias de cada variável, empregou-se o teste t de Student para a comparação entre médias, e o teste de qui-quadrado para avaliar a relação entre as variáveis de exposição e desfecho. Para a análise multivariável foi utilizada a regressão de Poisson com ajuste robusto da variância, para estimar a correlação entre o desfecho e as variáveis independentes. Para o cálculo das razões 
Modelo teórico proposto para investigação de condições associadas a prejuízo de desempenho em habilidades sociais entre usuários de crack. Região Metropolitana de Porto Alegre, Rio Grande do Sul, Brasil, 2011 (N = 519).

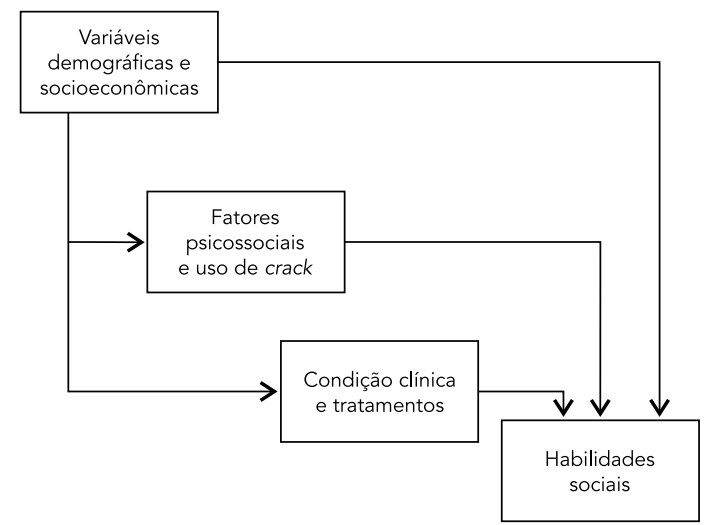

de prevalência ajustadas foram incluídas na análise as variáveis independentes de um mesmo nível hierárquico que estivessem em associação com o desfecho, na análise bruta, em nível de até $20 \%$ ( $\mathrm{p} \leq 0,20)$, mantendo-se no modelo as variáveis de níveis superiores que apresentaram associação estatisticamente significativa com o desfecho no ajuste para o seu nível. Foram consideradas significativas as associações com nível de significância de até $5 \%(\mathrm{p}<0,05)$.

\section{Resultados}

A amostra foi composta por 519 entrevistados, sendo 497 (95,8\%) homens e $22(4,2 \%)$ mulheres, com média de idades igual a 27,9 anos (desvio padrão - DP = 7,3 anos; intervalo de 95\% de confiança - IC95\%: 27,3-28,6). Mais da metade dos entrevistados ( $n=310 ; 59,7 \%$ ) informaram estar sem consumir crack por 12 semanas ou mais (o intervalo desde o último episódio de consumo variou de 85 dias a mais de 3 anos, com mediana igual 180 dias) e 209 (40,3\%) estavam sem usálo há menos de 84 dias (variando de menos de um dia a 14 dias, com mediana de 7 dias desde o último episódio).

A distribuição dos usuários de crack entrevistados quanto ao escore obtido no IHS mostrou 52,8 \% $(\mathrm{n}=274)$ deles apresentando escore igual ou inferior ao percentil 36, considerado deficitário. Não foram detectadas diferenças significativas entre os grupos quanto a estar ou não em atendimento no momento da entrevis- ta $(p=0,264)$. As Tabelas 1,2 e 3 mostram essa distribuição segundo as variáveis incluídas na análise em cada um dos níveis propostos no modelo hierarquizado.

A Tabela 1 apresenta a distribuição dos entrevistados quanto ao escore no IHS segundo as variáveis demográficas e socioeconômicas. Não foram consideradas para a estimativa de razões de prevalência as variáveis: sexo $(p=0,254)$, idade $(p=0,766)$, situação conjugal $(p=0,596)$, renda $(p=0,779)$ ou ocupação regular $(p=0,791)$. Número de pessoas no domicílio apareceu em associação com o escore deficitário no IHS ( $\mathrm{p}=$ 0,045 ) e a escolaridade do entrevistado apareceu em associação inversa ao desfecho ( $\mathrm{p}<0,001)$.

A Tabela 2 apresenta a distribuição dos entrevistados quanto ao escore no IHS segundo fatores psicossociais estudados e variáveis relacionadas ao uso do crack. Percebe-se que o déficit no IHS não aparece associado ao relato de ter vivenciado luto de amigo ou amiga nos 12 meses que antecederam as entrevistas $(p=0,155)$, nem com passagem por desemprego ou falência ( $\mathrm{p}=$ 0,927 ), ou separação conjugal no período de um ano ( $p=0,557)$. A comparação entre o grupo de usuários considerados abstinentes (sem consumir crack por um período mínimo de 12 semanas) e aqueles em uso ativo também não evidenciou diferença significativa quanto ao desfecho ( $p=0,906$ ). O uso de crack compartilhado com familiares também não guardou associação com o desfecho em análise, nem quando questionado em relação às mães dos entrevistados $(p=0,148)$ nem com os pais $(\mathrm{p}=0,578)$ ou com irmãos $(\mathrm{p}=$ 
Distribuição de frequência dos usuários de crack entrevistados quanto ao escore no Inventário de Habilidades Sociais (IHS), segundo variáveis demográficas e socioeconômicas. Região Metropolitana de Porto Alegre, Rio Grande do Sul, Brasil, 2011 ( $\mathrm{N}=519)$.

\begin{tabular}{|c|c|c|c|c|}
\hline \multirow[t]{2}{*}{ Variáveis } & \multirow[t]{2}{*}{$\mathrm{n}$} & \multicolumn{2}{|c|}{ Escore no IHS } & \multirow[t]{2}{*}{ Valor de $p$} \\
\hline & & Normal (\%) & Deficitário (\%) & \\
\hline Sexo & & & & 0,254 \\
\hline Masculino & 497 & $232(46,7)$ & $265(53,3)$ & \\
\hline Feminino & 22 & $13(59,1)$ & $9(40,9)$ & \\
\hline Idade (em anos completos) & & & & 0,766 \\
\hline $12-17$ & 18 & $10(55,6)$ & $8(44,4)$ & \\
\hline $18-30$ & 342 & $161(47,1)$ & $181(52,9)$ & \\
\hline 30 ou mais & 159 & $74(46,5)$ & $85(53,5)$ & \\
\hline Situação conjugal & & & & 0,596 \\
\hline Com companheiro/a & 109 & $49(45,0)$ & $60(55,0)$ & \\
\hline Sem companheiro/a & 410 & $196(47,8)$ & $214(52,2)$ & \\
\hline Número de pessoas no domicílio & & & & 0,045 \\
\hline Até 3 & 311 & $158(50,8)$ & $153(49,2)$ & \\
\hline 4 ou mais & 208 & $87(41,8)$ & $121(58,2)$ & \\
\hline Escolaridade (em anos completos) & & & & $<0,001$ \\
\hline Até 8 & 305 & $124(40,7)$ & $181(59,3)$ & \\
\hline 9 ou mais & 214 & $121(56,5)$ & $93(43,5)$ & \\
\hline Renda (salário mínimo nacional) & & & & 0,779 \\
\hline Sem renda no momento & 220 & $105(47,7)$ & $115(52,3)$ & \\
\hline Até 2 & 210 & $101(48,1)$ & $109(51,9)$ & \\
\hline Acima de 2 & 89 & $39(43,8)$ & $50(56,2)$ & \\
\hline Mantém ocupação regular? & & & & 0,791 \\
\hline Não & 232 & $108(46,6)$ & $124(53,4)$ & \\
\hline Sim & 285 & $136(47,7)$ & $149(52,3)$ & \\
\hline
\end{tabular}

$0,335)$. Entre essas variáveis, o relato de luto $(\mathrm{p}=$ $0,155)$ e já ter fumado crack com a mãe ( $\mathrm{p}=$ 0,148 ) preencheram o critério de associação com o desfecho com $\mathrm{p} \leq$ 0,20 e passaram às análises subsequentes, assim como o registro de luto de filho ou filha ( $p=0,032$ ), que esteve associado ao desfecho, e o apoio social percebido pelos entrevistados $(\mathrm{p}<0,001)$ e seu nível de resiliência $(\mathrm{p}<0,001)$, que apareceram em associação inversa ao registro de escore deficitário no IHS.

A Tabela 3 mostra a distribuição dos entrevistados quanto ao escore no IHS segundo as variáveis que descrevem a sua condição clínica naquele momento, o histórico de tratamentos e danos percebidos pelo entrevistado como decorrentes do uso do crack. Nesse grupo de variáveis, a que reunia critérios para dependência do crack ( $\mathrm{p}=0,920$ ) não prosseguiu para as etapas subsequentes de análise. Entre as que permaneceram no modelo não se verificou associação significativa entre o desfecho e as variáveis: afastamento de amigos ou grupo social $(\mathrm{p}=0,185)$, perda de contato com os pais ( $p=0,068)$, o desejo de cessar o uso de outras drogas, além do $\operatorname{crack}(\mathrm{p}=$ $0,058)$ e já ter ficado sem comer para obter a droga $(p=0,082)$. Associados ao escore deficitário no IHS foram encontrados: o desejo de cessar o uso de álcool, além do $\operatorname{crack}(\mathrm{p}=0,016)$, o desejo de cessar o uso de maconha, além do $\operatorname{crack}$ ( $\mathrm{p}=$ 0,029 ), o escore indicativo de sintomatologia depressiva do BDI ( $p=0,002)$, o escore indicativo de sintomatologia ansiosa do BAI $(p=0,017)$ e o relato de já ter assaltado ou furtado para obter a droga ( $p=0,021)$. Em associação inversa ao escore no IHS apareceram: o escore no URICA, com maior concentração de indivíduos com escore deficitário no IHS entre os que se apresentavam em estágios de pré-contemplação ou contemplação no URICA ( $\mathrm{p}=0,018$ ); já ter sido hospitalizado em função do uso de $\operatorname{crack}(\mathrm{p}=0,028)$ e o relato de já ter mantido relações sexuais para obter a droga $(\mathrm{p}=0,039)$. 
Tabela 2

Distribuição de frequência dos usuários de crack entrevistados quanto ao escore no Inventário de Habilidades Sociais (IHS), segundo fatores psicossociais e variáveis relacionadas ao uso da droga. Região Metropolitana de Porto Alegre, Rio Grande do Sul, Brasil, 2011 (N = 519 ).

\begin{tabular}{|c|c|c|c|c|}
\hline \multirow[t]{2}{*}{ Variáveis } & \multirow[t]{2}{*}{$\mathrm{n}$} & \multicolumn{2}{|c|}{ Escore no IHS } & \multirow[t]{2}{*}{ Valor de $p$} \\
\hline & & Normal (\%) & Deficitário (\%) & \\
\hline Apoio social (em tercis) & & & & $<0,001$ \\
\hline Inferior & 174 & $58(33,3)$ & $116(66,7)$ & \\
\hline Intermediário & 170 & $80(47,1)$ & $90(52,9)$ & \\
\hline Elevado & 174 & $107(61,5)$ & $67(38,5)$ & \\
\hline Resiliência (em tercis) & & & & $<0,001$ \\
\hline Inferior & 170 & $60(35,3)$ & $110(64,7)$ & \\
\hline Intermediário & 176 & $79(44,9)$ & $97(55,1)$ & \\
\hline Elevado & 171 & $105(61,4)$ & $66(38,6)$ & \\
\hline Já viveu luto de filho/a & & & & 0,032 * \\
\hline Não & 513 & $151(49,8)$ & $152(50,2)$ & \\
\hline Sim & 6 & $0(0,0)$ & $6(100,0)$ & \\
\hline Já viveu luto de amigo/a & & & & 0,155 \\
\hline Não & 303 & $245(47,8)$ & $268(52,2)$ & \\
\hline Sim & 216 & $94(43,5)$ & $122(56,5)$ & \\
\hline Desemprego ou falência nos últimos 12 meses & & & & 0,927 \\
\hline Não & 232 & $109(47,0)$ & $123(53,0)$ & \\
\hline Sim & 287 & $136(47,4)$ & $151(52,6)$ & \\
\hline Separação conjugal nos últimos 12 meses & & & & 0,557 \\
\hline Não & 293 & $135(46,1)$ & $158(53,9)$ & \\
\hline Sim & 226 & $110(48,7)$ & $116(51,3)$ & \\
\hline Condição de uso nas últimas 12 semanas & & & & 0,906 \\
\hline Ativo & 209 & $98(46,9)$ & $111(53,1$ & \\
\hline Abstinente & 310 & $147(47,4)$ & $163(52,6)$ & \\
\hline Já fumou crack com a mãe & & & & 0,148 \\
\hline Não & 466 & $215(46,1)$ & $251(53,9)$ & \\
\hline Sim & 53 & $30(56,6)$ & $23(43,4)$ & \\
\hline Já fumou crack com o pai & & & & 0,578 \\
\hline Não & 475 & $222(46,7)$ & $253(53,3)$ & \\
\hline Sim & 43 & $22(51,2)$ & $21(48,8)$ & \\
\hline Já fumou crack com irmãos & & & & 0,335 \\
\hline Não & 386 & $187(48,5)$ & $199(51,5)$ & \\
\hline Sim & 133 & $58(43,6)$ & $75(56,4)$ & \\
\hline
\end{tabular}

* Valor de p para teste exato de Fisher.

A Tabela 4 mostra as razões de prevalências brutas e ajustadas para o escore deficitário no IHS, segundo cada uma das variáveis de exposição selecionadas. Não se mantiveram associadas ao escore deficitário no IHS as variáveis: número de pessoas no domicílio ( $p=0,123$ ), ter vivido luto de amigo ( $\mathrm{p}=0,304)$, já ter fumado crack com a mãe $(p=0,055)$, estar afastado de amigos ou grupo social em função do uso de $\operatorname{crack}$ ( $\mathrm{p}=$ $0,101)$, desejar cessar o uso de outras drogas $(\mathrm{p}=$ $0,910)$, álcool ( $\mathrm{p}=0,662)$ ou maconha ( $\mathrm{p}=0,313)$, além do crack, ter sintomas ansiosos no escore do BAI ( $\mathrm{p}=0,890$ ) e já ter ficado sem comer para obter o $\operatorname{crack}(\mathrm{p}=0,232)$.

A Tabela 4 mostra ainda que tem probabilidade aumentada de ocorrência do escore deficitário no IHS os usuários de crack que obtiveram escores no URICA compatíveis com estágios de pré-contemplação ou contemplação, em termos de motivação para a mudança de comportamento relacionado ao crack $(\mathrm{RP}=1,18$; IC95\%: $1,02-1,38 ; p=0,031)$, aqueles que atingiram escores mais elevados no BDI - identificados como tendo sintomas depressivos $(\mathrm{RP}=1,21$; IC95\%: 
Tabela 3

Distribuição de frequência dos usuários de crack entrevistados quanto ao escore no Inventário de Habilidades Sociais (IHS), segundo sua condição clínica, tratamentos e danos pelo uso da droga. Região Metropolitana de Porto Alegre, Rio Grande do Sul, Brasil, 2011 (N = 519 ).

\begin{tabular}{|c|c|c|c|c|}
\hline \multirow[t]{2}{*}{ Variáveis } & \multirow[t]{2}{*}{$\mathbf{n}$} & \multicolumn{2}{|c|}{ Escore no IHS } & \multirow[t]{2}{*}{ Valor de $\mathrm{p}$} \\
\hline & & Normal (\%) & Deficitário (\%) & \\
\hline Preenche critérios para dependência & & & & 0,920 \\
\hline Não & 27 & $13(48,1)$ & $14(51,9)$ & \\
\hline Sim & 492 & $232(47,1)$ & $260(52,9)$ & \\
\hline Afastou-se de amigos ou grupo social em função do uso do crack & & & & 0,185 \\
\hline Não & 45 & $17(37,8)$ & $28(62,2)$ & \\
\hline Sim & 474 & $228(48,1)$ & $246(51,9)$ & \\
\hline Perdeu contato com seus pais em função do uso do crack & & & & 0,068 \\
\hline Não & 274 & $119(43,4)$ & $155(56,6)$ & \\
\hline Sim & 245 & $126(51,4)$ & $119(48,6)$ & \\
\hline Deseja acessar outras drogas além do crack & & & & 0,058 \\
\hline Não & 388 & $193(49,7)$ & $195(50,3)$ & \\
\hline Sim & 125 & $50(40,0)$ & $75(60,0)$ & \\
\hline Deseja acessar o uso de álcool & & & & 0,016 \\
\hline Não & 397 & $199(50,1)$ & $198(49,9)$ & \\
\hline Sim & 122 & $46(37,7)$ & $76(62,3)$ & \\
\hline Deseja acessar o uso de maconha & & & & 0,029 \\
\hline Não & 413 & $205(49,6)$ & $208(50,4)$ & \\
\hline Sim & 106 & $40(37,7)$ & $66(62,3)$ & \\
\hline Em pré-contemplação ou contemplação (URICA) & & & & 0,018 \\
\hline Não & 324 & $166(51,2)$ & $158(48,8)$ & \\
\hline Sim & 195 & $79(40,5)$ & $116(59,5)$ & \\
\hline Já foi hospitalizado pelo uso de crack & & & & 0,0028 \\
\hline Não & 270 & $115(42,6)$ & $155(57,4)$ & \\
\hline $\operatorname{Sim}$ & 249 & $130(52,2)$ & $119(47,8)$ & \\
\hline Sintomas depressivos segundo o BDI & & & & 0,002 \\
\hline Não & 247 & $134(54,2)$ & $113(45,8)$ & \\
\hline Sim & 272 & $111(40,8)$ & $161(59,2)$ & \\
\hline Sintomas ansiosos segundo o BAl & & & & 0,017 \\
\hline Não & 287 & $149(51,9)$ & $138(48,1)$ & \\
\hline Sim & 232 & $96(41,4)$ & $136(58,6)$ & \\
\hline \multicolumn{5}{|l|}{ Para obter o crack você já } \\
\hline Assaltou ou furtou? & & & & 0,021 \\
\hline Não & 254 & $133(52,4)$ & $121(47,6)$ & \\
\hline $\operatorname{Sim}$ & 265 & $112(42,3)$ & $153(57,7)$ & \\
\hline Manteve relações sexuais? & & & & 0,039 \\
\hline Não & 382 & $170(44,5)$ & $212(55,5)$ & \\
\hline Sim & 137 & $75(54,7)$ & $62(45,3)$ & \\
\hline Ficou sem comer? & & & & 0,082 \\
\hline Não & 104 & $57(54,8)$ & $47(45,2)$ & \\
\hline Sim & 415 & $188(45,3)$ & $227(54,7)$ & \\
\hline
\end{tabular}

BAI: Inventário de Ansiedade de Beck; BDI: Inventário de Depressão de Beck; URICA: University of Rhode Island Change Assessment.

1,01-1,45; $\mathrm{p}=0,037)$, os que informaram ter vivido luto de filho ou filha ( $\mathrm{RP}=1,56$; IC95\%: 1,21-2,02; $\mathrm{p}=0,001)$ e aqueles que disseram já ter assaltado ou furtado alguém para obter o crack $(\mathrm{RP}=1,22$;
IC95\%: 1,03-1,45; $p=0,020$ ). Foi identificada probabilidade diminuída de ocorrência do desfecho entre os usuários de crack com escolaridade de 9 anos completos de estudos ou mais $(\mathrm{RP}=0,75$; 
Tabela 4

Razões de prevalência (RP) e intervalos de 95\% de confiança (IC95\%) para Inventário de Habilidades Sociais (IHS) deficitário, segundo variáveis de exposição entre usuários de crack. Região Metropolitana de Porto Alegre, Rio Grande do Sul, Brasil, 2011 (N = 519).

\begin{tabular}{|c|c|c|c|c|}
\hline \multirow[t]{2}{*}{ Variáveis } & \multicolumn{2}{|c|}{ Análise bruta * } & \multicolumn{2}{|c|}{ Análise ajustada * } \\
\hline & RP & IC95\% & RP & IC95\% \\
\hline \multicolumn{5}{|l|}{ Nível distal } \\
\hline \multicolumn{5}{|l|}{ Número de pessoas no domicílio } \\
\hline Até 3 & 1,00 & & 1,00 & \\
\hline 4 ou mais & 1,18 & $1,01-1,39$ & 1,14 & $0,97-1,33$ \\
\hline \multicolumn{5}{|l|}{ Escolaridade (em anos completos) } \\
\hline$\leq 8$ & 1,00 & & 1,00 & \\
\hline$>8$ & 0,73 & $0,61-0,88$ & 0,75 & $0,62-0,89$ \\
\hline \multicolumn{5}{|l|}{ Nível intermediário } \\
\hline \multicolumn{5}{|l|}{ Apoio social (em tercis) } \\
\hline Inferior & 1,00 & & 1,00 & \\
\hline Intermediário & 0,79 & $0,67-0,95$ & 0,81 & $0,69-0,97$ \\
\hline Elevado & 0,58 & $0,47-0,72$ & 0,64 & $0,52-0,79$ \\
\hline \multicolumn{5}{|l|}{ Resiliência (em tercis) } \\
\hline Inferior & 1,00 & & 1,00 & \\
\hline Intermediário & 0,85 & $0,72-1,01$ & 0,90 & $0,76-1,07$ \\
\hline Elevado & 0,60 & $0,48-0,74$ & 0,63 & $0,51-0,78$ \\
\hline \multicolumn{5}{|l|}{ Já viveu luto de amigo/a } \\
\hline Não & 1,00 & & 1,00 & \\
\hline Sim & 1,13 & $0,96-1,32$ & 1,08 & $0,92-1,26$ \\
\hline \multicolumn{5}{|l|}{ Já viveu luto de filho/a } \\
\hline Não & 1,00 & & 1,00 & \\
\hline Sim & 1,91 & $1,76-2,08$ & 1,56 & $1,21-2,02$ \\
\hline \multicolumn{5}{|l|}{ Já fumou crack com a mãe } \\
\hline Não & 1,00 & & 1,00 & \\
\hline Sim & 0,81 & $0,59-1,11$ & 0,74 & $0,55-1,00$ \\
\hline \multicolumn{5}{|l|}{ Nível proximal } \\
\hline \multicolumn{5}{|c|}{ Afastou-se de amigos/grupo social por usar crack } \\
\hline Não & 1,00 & & 1,00 & \\
\hline Sim & 0,83 & $0,65-1,06$ & 0,81 & $0,62-1,04$ \\
\hline \multicolumn{5}{|l|}{ Perdeu contato com os pais por usar crack } \\
\hline Não & 1,00 & & 1,00 & \\
\hline Sim & 0,86 & $0,73-1,01$ & 0,84 & $0,71-0,98$ \\
\hline \multicolumn{5}{|l|}{ Deseja cessar outras drogas além do crack } \\
\hline Não & 1,00 & & 1,00 & \\
\hline Sim & 1,19 & $1,00-1,42$ & 0,98 & $0,67-1,43$ \\
\hline \multicolumn{5}{|l|}{ Deseja cessar o uso de álcool } \\
\hline Não & 1,00 & & 1,00 & \\
\hline Sim & 1,25 & $1,05-1,48$ & 1,07 & $0,80-1,42$ \\
\hline \multicolumn{5}{|l|}{ Deseja cessar o uso de maconha } \\
\hline Não & 1,00 & & 1,00 & \\
\hline $\operatorname{Sim}$ & 1,24 & $1,04-1,47$ & 1,17 & $0,86-1,60$ \\
\hline \multicolumn{5}{|l|}{ Estágio de motivação segundo URICA } \\
\hline Ação ou manutenção & 1,00 & & 1,00 & \\
\hline Pré-contemplação ou contemplação & 1,22 & $1,04-1,43$ & 1,18 & $1,02-1,38$ \\
\hline
\end{tabular}

(continua) 
Tabela 4 (continuação)

\begin{tabular}{|c|c|c|c|c|}
\hline \multirow[t]{2}{*}{ Variáveis } & \multicolumn{2}{|c|}{ Análise bruta * } & \multicolumn{2}{|c|}{ Análise ajustada * } \\
\hline & RP & IC95\% & RP & IC95\% \\
\hline \multicolumn{5}{|c|}{ Já foi hospitalizado pelo uso do crack } \\
\hline Não & 1,00 & & 1,00 & \\
\hline Sim & 0,83 & $0,70-0,98$ & 0,81 & $0,68-0,95$ \\
\hline \multicolumn{5}{|c|}{ Sintomas depressivos segundo o BDI } \\
\hline Não & 1,00 & & 1,00 & \\
\hline Sim & 1,29 & $1,09-1,53$ & 1,21 & $1,01-1,45$ \\
\hline \multicolumn{5}{|c|}{ Sintomas ansiosos segundo o BAl } \\
\hline Não & 1,00 & & 1,00 & \\
\hline Sim & 1,22 & $1,04-1,43$ & 0,99 & $0,83-1,17$ \\
\hline \multicolumn{5}{|c|}{ Para obter o crack você já assaltou? } \\
\hline Não & 1,00 & & 1,00 & \\
\hline Sim & 1,21 & $1,03-1,43$ & 1,22 & $1,03-1,45$ \\
\hline \multicolumn{5}{|c|}{ Para obter o crack você já manteve relações sexuais? } \\
\hline Não & 1,00 & & 1,00 & \\
\hline Sim & 0,82 & $0,66-1,00$ & 0,77 & $0,63-0,94$ \\
\hline \multicolumn{5}{|c|}{ Para obter o crack você já ficou sem comer? } \\
\hline Não & 1,00 & & 1,00 & \\
\hline Sim & 1,21 & $0,96-1,52$ & 1,14 & $0,92-1,41$ \\
\hline
\end{tabular}

BAI: Inventário de Ansiedade de Beck; BDI: Inventário de Depressão de Beck; IC95\%: intervalo de 95\% de confiança; RP: razão de prevalência; URICA: Univesity of Rhode Island Change Assessment.

* Análise bruta e ajustada por regressão de Poisson robusta, mantendo-se as variáveis dos níveis anteriores associadas em $p \leq 0,05$ e as do nível, associadas em até $\mathrm{p} \leq 0,20$.

IC95\%: 0,62-0,89; $\mathrm{p}=0,001)$, percepção de apoio social elevado $(\mathrm{RP}=0,64$; IC95\%: $0,52-0,79 ; \mathrm{p}<$ $0,001)$, resiliência elevada $(\mathrm{RP}=0,63$; IC95\%: $0,51-0,78 ; \mathrm{p}<0,001)$, que referiram ter perdido o contato com os seus pais ( $\mathrm{RP}=0,84$; IC95\%: 0,71 0,$98 ; \mathrm{p}=0,032$ ), que já foram hospitalizados em função do uso do crack (RP = 0,81; IC95\%: 0,68$0,95 ; \mathrm{p}=0,009)$ e que referiram já ter mantido relação sexuais para obtê-lo $(\mathrm{RP}=0,77$; IC95\%: $0,63-0,94 ; \mathrm{p}=0,009$ ).

\section{Discussão}

A ocorrência de déficit em habilidades sociais entre os usuários de crack entrevistados parece elevada se comparada às evidências de outros estudos semelhantes com usuários de diferentes substâncias psicoativas 29,30. Este trabalho não testa isso, mas é possível imaginar que o consumo de crack tenha uma relação diferenciada com níveis de habilidades sociais entre os seus usuários, se comparados a indivíduos em outras situações. O registro de déficit em habilidades sociais tem sido confirmado em associação a diferentes condições. A literatura tem sugerido a associação de déficits em habilidade sociais a quadros de fo- bia social, ao consumo de maconha, de bebidas alcoólicas e ao início do uso de tabaco 8,9,29.

Neste grupo de entrevistados, não foi verificada diferença entre os grupos com e sem déficit em habilidades sociais quanto a estar em atendimento no momento da entrevista e nem quanto ao intervalo de tempo decorrido desde o último episódio de consumo do crack, sugerindo que o escore no IHS não modifica o vínculo dos entrevistados aos atendimentos e nem a probabilidade de permanecerem abstinentes da droga. Na Espanha, também não foram observadas diferenças quanto ao que os autores chamaram de funções sociais, entre os pacientes que haviam sofrido recaída e aqueles que se mantiveram abstinentes ao longo de três meses 31 . Já nos Estados Unidos, foi evidenciado um maior déficit no inventário de ajuste social entre os usuários de cocaína ativos no momento da internação para tratamento em comparação aos usuários em abstinência no mesmo momento do estudo 32 . Eventuais diferenças entre esses estudos podem decorrer de especificidades dos sujeitos avaliados ou de aspectos culturais. Entre essas especificidades, cita-se, por exemplo, que pode haver comprometimento mais significativo entre usuários de cocaína que chegam à 
hospitalização no momento em que ela ocorre, talvez mais como decorrência do padrão de uso mais recente. Mas, podem se dever ao acaso, em função de limitações do próprio desenho de cada um dos estudos.

As análises propostas neste trabalho indicam que usuários de crack com probabilidade aumentada de apresentar déficit em habilidades sociais são os que referiram já ter vivenciado a morte de filho ou filha, os que apresentaram escore no BDI compatível com a ocorrência de sintomas depressivos no momento das entrevistas, os que afirmaram já ter tido envolvimento em assaltos ou furtos e os que obtiveram escores no URICA compatíveis com estágios de motivação para a mudança de pré-contemplação e contemplação.

As duas primeiras condições, luto por morte de filho e sintomas depressivos, estão potencialmente relacionadas uma com a outra e ambas são, frequentemente, determinantes de embotamento e retraimento social significativos. Esses sintomas são caracterizados por inibição comportamental, que contribui consideravelmente para a deterioração do funcionamento social. Embotamento e retraimento social se expressam no inventário de habilidades sociais contribuindo para a formação de escores inferiores, o que torna este achado, de certa forma, previsível 33,34,35. O falecimento de filhos é descrito como um evento capaz de gerar queixas emocionais e repercussões de cunho comportamental duradouras. Os relatos de sofrimento de pais e mães diante do falecimento de filhos e filhas de todas as idades descrevem o fato como um fenômeno devastador 34 . O luto parental costuma tomar proporções de sofrimento muito intensas, afetando, além da esfera individual, o relacionamento com parceiros e a dimensão social 36. De forma semelhante, os sintomas depressivos repercutem agudamente 18 .

O uso de crack aumenta o risco de ocorrência de sintomas depressivos, da mesma forma que o início do consumo de substâncias pode estar relacionado ao alívio da dor e do sofrimento 15,31,37,38. Um estudo brasileiro com usuários de cocaína, crack e álcool encontrou prevalência significativa de transtornos de humor como comorbidade nos quadros de dependência estudados, sendo a ocorrência de episódio depressivo maior bastante frequente na amostra 38. Níveis mais elevados de sintomatologia depressiva costumam ser descritos como associados a maior risco de suicídio, que ocorre mais entre usuários de substâncias psicoativas 38,39,40. A constatação de que usuários de crack com sintomas depressivos têm chance aumentada de ter habilidades sociais diminuídas torna a questão ainda mais preocupante. A dificuldade de se inserir ou de manter vínculos com grupos de apoio no meio social, pode promover uma redução ainda maior de alternativas para lidar com os desafios aos quais se veem expostos os indivíduos que fazem uso de $\mathrm{cra}$ ck. Corroborando essa ideia, aparece o achado de que o apoio social mais elevado, segundo a percepção do entrevistado, está associado com a menor probabilidade de ocorrer déficit de habilidades sociais.

O envolvimento em assaltos ou furtos provavelmente está associado ao desfecho proposto por representar uma forma mais drástica, imediata e menos elaborada do ponto de vista social de lidar com a insuficiência de recursos para obter a droga 41 . O envolvimento em furtos e assaltos tende a ser mais evidente entre os usuários de crack do que na população em geral, e está descrito como associado à necessidade de repetir o consumo, que nesta substância, tende a se mostrar de forma intensa e quase instantânea 18. Os dados deste trabalho mostram que ele tende a ser maior ainda entre usuários de crack com desempenho deficitário no IHS. O acesso ao crack se dá em pontos de tráfico, de forma ilícita, e com maior estímulo e aproximação às condições para o envolvimento em atos contrários à lei. Mesmo que estudos transversais, como este, sejam imprecisos quanto à determinação de causalidade 41 , é bastante plausível que soluções com perfil mais imediato e com recurso à força sejam preferidas por pessoas com repertórios de habilidades sociais mais limitados 42 . Além disso, não apresentar déficit em habilidades sociais parece proporcionar alternativas em situações de exposição social para obter o crack. Relações sexuais para obter a droga, ao contrário dos assaltos, foram mais referidas por entrevistados com desempenho não deficitário. Esses indivíduos tendem a se expressar de forma mais assertiva, manter maior contato visual, sorrir com mais frequência e manter uma fala com menos perturbações além de ter expectativas e reações mais positivas a situações sociais 2 . Supõe-se aqui que essas habilidades dão ao usuário um maior poder de sedução e convencimento, o que pode estar associado à constatação de que a atividade sexual garante o acesso ao crack.

Em relação aos estágios motivacionais, aferidos pelo URICA, a pré-contemplação é caracterizada pela ausência de reconhecimento da existência de um problema relacionado ao uso de substâncias, e a contemplação é caracterizada pelo reconhecimento da existência de um problema, porém com sentimentos ambivalentes em relação a manter ou cessar o uso de substâncias. Os terceiro e quarto estágios, ação e manutenção, estão relacionados, respectivamente, 
ao comprometimento com a mudança de comportamentos vinculados ao uso de drogas e à tomada de decisão de manter comportamentos abstinentes, uma vez alcançados 25. Levandose em conta as evidências que indicam um repertório diminuído de habilidades sociais como associado à iniciação e à manutenção do uso de substâncias, inclusive como alternativa de enfrentamento de estressores externos 4,5,6,7, o resultado encontrado, que sugere uma associação entre prejuízos nas habilidades sociais de usuários de crack e ausência de engajamento em comportamentos direcionados à mudança e abstinência do uso da droga, pode ser considerado dentro do esperado. Da mesma forma, a menor probabilidade de déficit de habilidades sociais verificada entre os usuários que referiram já ter buscado hospitalização por problemas decorrentes do uso de crack, parece indicar que o maior desenvolvimento das habilidades sociais entre estes usuários pode favorecer movimentos no sentido de buscar atendimento e empenharse em promover alguma mudança em sua condição, melhorando a expectativa de resposta aos planos de tratamento $11,12,13,14$. Nenhuma diferença significativa, no entanto, foi encontrada para a ocorrência do desfecho em análise entre os grupos de entrevistados que estavam ou não em tratamento, nem quanto ao tempo decorrido desde o último episódio de uso da droga. O efeito do desenvolvimento de habilidades sociais pode não ser tão abrangente nesse sentido ou outras condições, que precisariam ser mais bem estudadas, podem estar aí interferindo.

Os resultados também demonstraram que o elevado apoio social está associado às habilidades sociais. Entende-se que, nesse caso, é provável que esse apoio tenha sido conquistado por meio das relações estabelecidas pelos sujeitos que dispõem deste recurso e, ao contrário, os usuários de crack com déficit de habilidades sociais têm menos apoio possivelmente pela sua dificuldade em desenvolver e estabelecer relações que lhes tragam suporte. Indivíduos que apresentam um melhor repertório de habilidades sociais tendem a ter um desempenho socialmente mais competente. Esse desempenho é possível mediante a capacidade de uma leitura adequada do contexto social, com boa compreensão dos desempenhos efetivos e esperados para o sujeito ao se relacionar com os outros 43 . Essa leitura efetiva do ambiente social e o emprego adequado das habilidades disponíveis possibilitam o estabelecimento de um maior número de vínculos e inserção em grupos, o que leva ao maior apoio social.

Do mesmo modo, ocorre maior resiliência entre os usuários com menor déficit em habi- lidades sociais, pois estes sujeitos dispõem de mais recursos para lidar com frustrações e maior capacidade de aprendizado nas interações. Além disso, a literatura aponta para a competência social como um fator de proteção ao indivíduo em diversos processos adaptativos, possibilitando bons resultados em diversas situações e evitando uma série de efeitos negativos, o que contribui para resiliência 44 .

Os resultados também mostraram que quando o nível de escolaridade foi maior, o escore de déficit de habilidades sociais foi menor, o que leva a crer que a escola pode ser um ambiente de desenvolvimento supervisionado destas habilidades. A escola envolve uma série de interações contínuas entre muitas pessoas com diferentes características, configurando-se como um ambiente que, além de processos de aprendizagem, abrange a construção de laços afetivos e a preparação para a integração na sociedade. O desenvolvimento e estabelecimento desses laços possibilitam que os indivíduos aprendam a lidar com conflitos, capacitando-os a resolver problemas tanto de forma conjunta como individual 45 .

Destacou-se, ainda, que escore deficitário de habilidades sociais esteve associado à menor probabilidade de registro de perda de contato com os pais em função do uso de crack. Sugerese que dispor de maiores recursos de habilidades sociais pode facilitar a adaptação do usuário fora do ambiente familiar, por apresentar maior autonomia e resiliência, menor dependência desta relação e maior rede de relações (apoio social) que o ajudam a se manter, mesmo afastado dos pais. Essa evidência é, em parte, conflitante com a ideia de que as habilidades sociais estão associadas ao melhor uso de estratégias de resolução de conflitos ou de dificuldades 46. Além disso, indivíduos com menor déficit em habilidades sociais tendem a ter pais mais habilidosos socialmente, visto que, em grande medida, o ambiente familiar é o primeiro promotor de comportamentos socialmente adequados, bem como mantenedor destes comportamentos. Para que os filhos tenham comportamentos adequados, é necessário que os pais comportem-se de maneira socialmente adequada, servindo de modelo e utilizando de reforçadores dos comportamentos desejáveis dos filhos 42 . Pais habilidosos socialmente apresentam padrões comportamentais e cognitivos que constituem respostas adequadas às mais diversas situações 47 . Isso leva a crer que, diante das condições críticas relacionadas ao consumo de crack, por apresentarem mais recursos, ocorram mais tensões no grupo familiar e mais rupturas. Abrir mão da relação que se torna exaustivamente conflituosa é mais viável para quem tem mais recursos pessoais e sociais. 
São limitações deste estudo o processo amostral, potencialmente determinante de viés de seleção, e o fato de que muitos dos dados analisados são obtidos por recordatório, potencialmente submetidos a viés de informação 48,49 . Cabe destacar, porém, que o número de entrevistados é suficiente para garantir precisão e poder estatístico às análises realizadas, e os grupos com e sem déficit de habilidades sociais são semelhantes quanto a sexo, idade, estado civil, renda e ocupação. $\mathrm{O}$ viés de informação é controlado pela repetição das orientações, com indicação clara dos intervalos de tempo aos quais se referiam cada uma das perguntas que dependiam de recordatório, e com o estímulo ao entrevistado para que buscasse as informações calmamente, sendo o risco homogêneo entre estudos semelhantes 50,51 .

Os resultados encontrados neste trabalho possibilitaram apresentar algumas condições associadas a escores deficitários no IHS entre usuários de crack da Região Metropolitana de Porto Alegre. Não há como precisar se as condições associadas ao escore deficitário no inventário são prévias ou decorrentes do uso do crack, porém conhecê-las possibilita uma melhor compreensão do perfil desses usuários.

Apesar do escore deficitário ou não no inventário não ser determinante de adesão ao tratamento ou abstinência, um maior repertório de habilidades sociais amplia a rede de apoio, aspecto importante no tratamento desses usuários. Ainda que a atividade sexual para obter a droga e ruptura de laços familiares não sejam opções recomendáveis, o menor envolvimento em assaltos e situações de violência é um benefício relevante e atribuível à qualificação em termos de habilidades sociais.

Todos os achados do estudo reforçam a importância de controle das variáveis em trabalhos futuros sobre o tema, além de evidenciarem a relevância da inclusão da avaliação de habilidades sociais no ingresso de usuários de crack em centros de atendimento e da individualização do planejamento terapêutico, de modo a contemplar estes diferentes perfis de comprometimento pessoal e social associados ao uso do crack.

\section{Colaboradores}

R. L. Horta coordenou o projeto original, a coleta e análise de dados, e aprovou a versão final do manuscrito. J. L. Schäfer participou da análise dos dados, da revisão da literatura e respondeu por parte da redação da versão final do manuscrito. L. R. M. Coelho colaborou na análise de dados e na revisão da literatura, redigiu parte da versão final do manuscrito e o aprovou na íntegra. V. S. Rodrigues colaborou no desenvolvimento do projeto, treinamento da equipe de campo, revisou a análise de dados e aprovou a versão final do manuscrito. M. S. Oliveira colaborou no desenvolvimento do projeto, revisão da literatura e aprovou a versão final do manuscrito. V. A. Teixeira colaborou na análise de dados, revisão da literatura e contribuiu na redação da versão final do manuscrito. 
1. Bolsoni-Silva AT. Habilidades sociais: breve análise da teoria e da prática à luz da análise do comportamento. Interação em Psicologia 2002; 6:233-42.

2. Caballo VE. Diferenças entre indivíduos socialmente hábeis e não-hábeis. In: Caballo VE, organizador. Manual de avaliação e treinamento das habilidades sociais. São Paulo: Editora Santos; 2012. p. 99-108.

3. Del Prette ZAP, Del Prette A. Habilidades sociais e análise do comportamento: proximidade histórica e atualidades. Revista Perspectivas 2010; 1:104-15.

4. Rice P, Caballo V. O enfrentamento do estresse: estratégias cognitivo-comportamentais. In: Caballo VE, organizador. Manual para o tratamento cognitivo-comportamental dos transtornos psicológicos da atualidade. São Paulo: Editora Santos; 2007. p. 301-36.

5. Maisto SA, Connors GJ, Zywiak WH. Alcohol treatment changes in coping skills, self-efficacy, and levels of alcohol use and related problems 1 year following treatment initiation. Psychol Addict Behav 2000; 14:257-66.

6. Barkin SL, Smith KS, DuRant RH. Social skills and attitudes associated with substance use behaviors among young adolescents. J Adolesc Health 2002; 30:448-54.

7. Wagner MF, Oliveira MS. Habilidades sociais e abuso de drogas em adolescentes. Psicol Clín 2007; 19:101-16.

8. Wagner MF, da Silva JG, Zanettelo LB, da Silva Oliveira M. O uso da maconha associado ao déficit de habilidades sociais em adolescentes. SMAD, Rev Eletrônica Saúde Mental Álcool Drog 2010; 6:255-73.

9. Rodrigues VS. Oliveira MS. Habilidades sociales y ansiedad social en fumadores y no fumadores. Psicol Conduct 2010; 18:183-95.

10. Aliane PP, Lourenço LM, Ronzani TM. Estudo comparativo das habilidades sociais de dependentes e não dependentes de álcool. Psicol Estud 2006; 11:83-8.

11. Bordin S, Zanelatto NA, Figlie NB, Laranjeira R. Treinamento de habilidades sociais e de enfrentamento de situações de risco. In: Figlie NB, Bordin $\mathrm{S}$, Laranjeira R, organizadores. Aconselhamento em dependência química. 2a Ed. São Paulo: Editora Roca; 2010. p. 313-54.

12. Caballo VE. El entrenamiento en habilidades sociales. In: Caballo VE, organizador. Manual de técnicas de terapia y modificación de conducta. 4a $\mathrm{Ed}$. Madrid: Siglo XXI; 1998.

13. Knapp P, Luz Júnior E. Terapia cognitivo-comportamental dos comportamentos adictivos. In: Cordioli AV, editor. Psicoterapias abordagens atuais. 3 a Ed. Porto Alegre: Editora Artmed; 2008. p. 616-40.

14. Monti PM, Kadden RM, Rohsenow DJ, Cooney NL. Tratando a dependência de álcool: um guia de treinamentos das habilidades de enfrentamento. São Paulo: Editora Roca; 2005.
15. Smith DC, Tabb KM, Fisher D, Cleeland L. Drug refusal skills training does not enhance outcomes of African American adolescents with substance use problems. J Subst Abuse Treat 2014; 46:274-9.

16. Duailibi LB, Ribeiro M, Laranjeira R. Profile of cocaine and crack users in Brazil. Cad Saúde Pública 2008; 24 Suppl 4:S545-57.

17. Ribeiro M, Dunn J, Laranjeira R, Sesso R. High mortality among young crack cocaine users in Brazil: a 5-year follow-up study. Addiction 2004; 99:1133-5.

18. Ribeiro M, Dunn J, Sesso R, Dias AC, Laranjeira R. Causes of death among crack cocaine users. Rev Bras Psiquiatr 2006; 28:196-202.

19. Guimarães CF, Santos DVV, Freitas RC, Araujo RB. Perfil do usuário de crack e fatores relacionados à criminalidade em unidade de internação para desintoxicação no Hospital Psiquiátrico São Pedro de Porto Alegre (RS). Rev Psiquiatr Rio Gd Sul 2008; 30:101-8.

20. Lejuez CW, Zvolensky MJ, Daughters SB, Bornovalova MA, Paulson A, Tull MT, et al. Anxiety sensitivity: a unique predictor of dropout among inner-city heroin and crack/cocaine users in residential substance use treatment. Behav Res Ther 2008; 46:811-8.

21. Levitan M, Rangé B, Nardu AE. Habilidades sociais na agorafobia e fobia social. Psicol Teor Pesqui 2008; $24: 95-9$

22. Cunha PJ, Nicastri S, Gomes LP, Moino RM, Peluso MA. Alterações neuropsicológicas em dependentes de cocaína/crack internados: dados preliminares. Rev Bras Psiquiatr 2004; 26:103-6.

23. Del Prette Z, Del Prette A. Inventário de habilidades sociais: manual de aplicação e interpretação. São Paulo: Casa do Psicólogo; 2001.

24. Pesce RP, Assis SG, Avanci JQ, Santos NC, Malaquias JV, Carvalhaes R. Adaptação transcultural, confiabilidade e validade da escala de resiliência. Cad Saúde Pública 2005; 21:436-48.

25. Del Rio Szupszynski KP, Oliveira MS. Adaptação brasileira da University of Rhode Island Change Assessment (URICA) para usuários de substâncias ilícitas. Psico-USF 2008; 13:31-9.

26. Beck AT, Ward CH, Mendelson M, Mock J, Erbaugh, J. An inventory for measuring depression. Arch Gen Psychiatry 1961; 4:561-71.

27. Cunha JA. Manual da versão em português das Escalas Beck. São Paulo: Casa do Psicólogo; 2001.

28. Hughes A, Sathe N, Spagnola K. State estimates of substance use from the 2006-2007 National Surveys on Drug Use and Health. Rockville: Substance Abuse and Mental Health Services Administration, U.S. Department of Health and Human Services; 2009. (NSDUH Series H-35, HHS Publication SMA 09-4362).

29. Cunha SM, Carvalho JCN, Kolling NM, Silva, CR, Kristensen $\mathrm{CH}$. Habilidades sociais em alcoolistas: um estudo exploratório. Rev Bras Ter Cogn 2007; 3:31-9. 
30. Del Prette Z, Del Prette A, Barreto MCM. Análise de um Inventário de Habilidades Sociais (IHS) em uma amostra de universitários. Psicol Teor Pesqui 1998; 14:219-28.

31. Verdejo-Garcia A, Albein-Urios N, Martinez-Gonzalez JM, Civit E, de la Torre R, Lozano O. Decisionmaking impairment predicts 3-month hair-indexed cocaine relapse. Psychopharmacology Berl) 2014; 231:4179-87.

32. Cochran G, Stitzer M, Nunes EV, Hu MC, Campbell A. Clinically relevant characteristics associated with early treatment drug use versus abstinence. Addict Sci Clin Pract 2014; 9:6.

33. Aho AL, Tarkka MT, Åstedt-Kurki P, Kaunonen M. Fathers' experience of social support after the death of a child. Am J Mens Health 2009; 3:93-103.

34. Meert KL, Donaldson AE, Newth CJ, Harrison R, Berger J, Zimmerman J, et al. Complicated grief and associated risk factors among parents following a child's death in the pediatric intensive care unit. Arch Pediatr Adolesc Med 2010; 164:1045-51.

35. Rebelo JE. Importância da entreajuda no apoio a pais em luto. Anál Psicol 2005; 23:373-80.

36. Bittencourt ALP, Quintana AM, Velho MTC. A perda do filho: luto e doação de órgãos. Estud Psicol (Campinas) 2011; 28:435-42.

37. Cruz M, Bertoni N, Bastos FI, Burnett C, Gooch J, Fischer B. Comparing key characteristics of young adult crack users in and out-of-treatment in Rio de Janeiro, Brazil. Subst Abuse Treat Prev Policy 2014; 9:2.

38. Scheffer M, Pasa GG, Almeida RMM. Dependência de álcool, cocaína e crack e transtornos psiquiátricos. Psicol Teor Pesqui 2010; 26:533-41.

39. Demetrovics Z. Co-morbidity of drug addiction: an analysis of epidemiological data and possible etiological models. Addict Res Theory 2009; 17:420-31.

40. Hess ARB, Almeida RMM, Moraes AL. Comorbidades psiquiátricas em dependentes químicos em abstinência em ambiente protegido. Estud Psicol (Natal) 2012; 17:171-8.
41. Morton WA. Cocaine and psychiatric symptoms. Prim Care Companion J Clin Psychiatry 1999; 1:109-13.

42. Clark CB, Reiland S, Thorne C, Cropsey KL. Relationship of trauma exposure and substance abuse to self-reported violence among men and women in substance abuse treatment. J Interpers Violence 2014; 29:1514-30.

43. Bolsoni-Silva AT, Maturano EM. Práticas educativas e problemas de comportamento: uma análise à luz das habilidades sociais. Estud Psicol (Natal) 2002; 7:227-35.

44. Cecconello AM, Koller SH. Competência social e empatia: um estudo sobre resiliência com crianças em situação de pobreza. Estud Psicol (Natal) 2000; 5:71-93.

45. Dessen MA, Polonia AC. A família e a escola como contextos de desenvolvimento humano. Paidéia 2007; 17:21-32.

46. Bolsoni-Silva AT, Carrara, K. Habilidades sociais e análise do comportamento: compatibilidades e dissensões conceitual-metodológicas. Psicol Rev (Belo Horizonte) 2010; 16:330-50.

47. Pacheco JTB, Teixeira MAP, Gomes WB. Estilos parentais e desenvolvimento de habilidades sociais. Psicol Teor Pesqui 1999; 15:117-26.

48. Jekel JF, Katz DL, Elmore JG. Epidemiologia, bioestatísitca e medicina preventiva. Porto Alegre: Editora Artes Médicas; 2005.

49. Santana VS, Cunha S. Estudos transversais. In: Barreto ML, Almeida Filho N, organizadores. Epidemiologia \& saúde: fundamentos, métodos e aplicações. Rio de Janeiro: Editora Guanabara Koogan; 2011. p. 186-93.

50. Pearce N. Classification of epidemiological study designs. Int J Epidemiol 2012; 41:393-7.

51. Rothman KJ, Gallacher JE, Hatch EE. Why representativeness should be avoided. Int J Epidemiol 2013; 42:1012-4. 


\section{Abstract}

The article analyzes conditions associated with low scores on the Social Skills Inventory (SSI) among crack users in Greater Metropolitan Porto Alegre, Rio Grande do Sul State, Brazil, based on a cross-sectional study in a convenience sample of 519 individuals interviewed in 2011. Low SSI scores occurred in 52.8\% of the sample, and there were no differences according to currently active use of crack (yes/no) or current addiction treatment. Higher likelihood of low SSI was associated with crack users in pre-contemplation or contemplation in University of Rhode Island Change Assessment - URICA $(p=0.031)$, high Beck Depression Inventory - BDI scores ( $p=0.037)$, mourning a deceased child ( $p=0.001)$, and having committed armed robbery to obtain crack $(p=0 / 020)$. Lower likelihood of low SSI was associated with 9 or more years of schooling ( $p=0.001$ ), high social support $(p<0.001)$, high resilience $(p<0.001)$, having lost contact with parents ( $p=0.032)$, history of hospitalization due to crack $(p=0.009)$, and history of sex to obtain crack $(p=0.009)$. The findings are clinically useful and highlight the issue's importance.

Social Skills; Crack; Cocaine; Emotional Intelligence; Substance-Related Disorders

\section{Resumen}

El artículo presenta las condiciones asociadas a marcadores deficitarios en el Inventario de Habilidades Sociales (IHS) entre usuarios de crack de la Región Metropolitana de Porto Alegre, Río Grande do Sul, Brasil. Se trata de un estudio transversal, con una muestra de conveniencia de 519 individuos, entrevistados en 2011. El marcador deficitario en el IHS se produjo en un $52,8 \%$ de la muestra y no hubo diferencias en cuanto a estar o no consumiendo activamente crack, o en cuanto a estar no bajo supervisión médica. Tuvieron una probabilidad mayor de déficit en el IHS los consumidores de crack pre-contemplados o contemplados en el University of Rhode Island Change Assessment - URICA $(p=0,031)$, con marcadores elevados en Beck Depression Inventory $-B D I(p=0,037)$, quienes vivieron luto por un hijo $(p=0,001)$ y los que ya robaron para obtener crack $(p=0,020)$. Con probabilidad disminuida estaban quienes tenían una escolaridad de 9 años o más $(p=0,001)$, apoyo social elevado $(p<0,001)$, resiliencia elevada ( $p<0,001)$, con historial de haber perdido el contacto con sus padres ( $p=0,032)$, ya habiendo sido hospitalizado por crack $(p=0,009)$ y que ya informaron haber mantenido relaciones sexuales para obtener crack ( $p=0,009)$. Los hallazgos tienen utilidad clínica y muestran la importancia del tema.

Habilidades Sociales; Crack; Cocaína; Inteligencia

Emocional; Trastornos Relacionados com Substancias 